\title{
Holistic University Website Management: People, Technology and Processes
}

\author{
Feras Al-Hawari
}

German Jordanian University, P.O. Box 35247, Amman, 11180, Jordan

\begin{abstract}
The fact that a university website is developed and managed primarily in-house entails addressing multiple challenges to enhance the product quality, organization productivity, and customer satisfaction. Therefore, this paper introduces a holistic approach to website management that incorporates three key elements of software management: people, technology, and processes. Accordingly, a multidisciplinary team structure with distinctly outlined leadership, roles, skills, and responsibilities is proposed to manage websites effectively. Besides, an appropriate technology stack is identified to develop a website that is content-based, has a flat content structure, is not interactive, and does not require complex workflows. Furthermore, suitable project management, software development, and website business processes are adopted to produce high-quality services while satisfying time and cost constraints. Finally, applicable evaluation methods and key performance indicators are suggested to assess the effectiveness of the adopted business processes and the quality attributes of the website.
\end{abstract}

Keywords - project management process, content management system, software development process, website business processes, Drupal.

\section{Introduction}

A university website is a front-facing site for marketing the university programs and services to

DOI: $10.18421 /$ TEM94-60

https://doi.org/10.18421/TEM94-60

Corresponding author: Feras Al-Hawari, German Jordanian University, Amman, 11180, Jordan. Email: firas.alhawari@gju.edu.jo

Received: 02 June 2020.

Revised: 02 November 2020.

Accepted: 09 November 2020.

Published: 27 November 2020.

(c) BY-NC-ND (C) 2020 Feras Al-Hawari; published by UIKTEN. This work is licensed under the Creative Commons Attribution-NonCommercial-NoDerivs 4.0 License.

The article is published with Open Access at www.temjournal.com many stakeholders like students, parents, alumni, partners, employees, and suppliers.

Besides, it is considered the primary channel to establish the online presence of an institution. Therefore, the management and development of such an important business channel demands special attention to attract the best talent to the university, and hence increase the chances of institutional success.

In that regard, most university websites are developed and hosted in-house for continual, speedy, and cost-effective customization that reflects the vision, values, strengths, and objectives of the institution. Thus, operating a high-quality website entails overcoming many challenges such as:

- Identifying the structure, roles, skills, and responsibilities of the team responsible for building and maintaining the website.

- Pinpointing the proper website development framework and the technology stack (i.e., operating system, web server, and database management system) that it runs on.

- Adopting appropriate project management and website development processes to deliver the required features with high quality and without violating the desired time and cost constraints.

- Ensuring the quality level of the website in terms of content, timeliness, structure, engagingness, attractiveness, consistency, ease of use, speed, availability, and security.

- Determining the services needed to keep the website in great shape, and then guaranteeing that those services are executed smoothly by the web team to enhance the website division productivity, and thus improve customer satisfaction.

Accordingly, building a high-quality website is directly interrelated as a whole to the success of the website projects and the website division. Therefore, the main focus of this work is on identifying the key elements of a holistic approach to the German Jordanian University (GJU) website management, which is also suitable to manage similar university websites. The proposed approach in Figure 1 is based on the following three elements: 


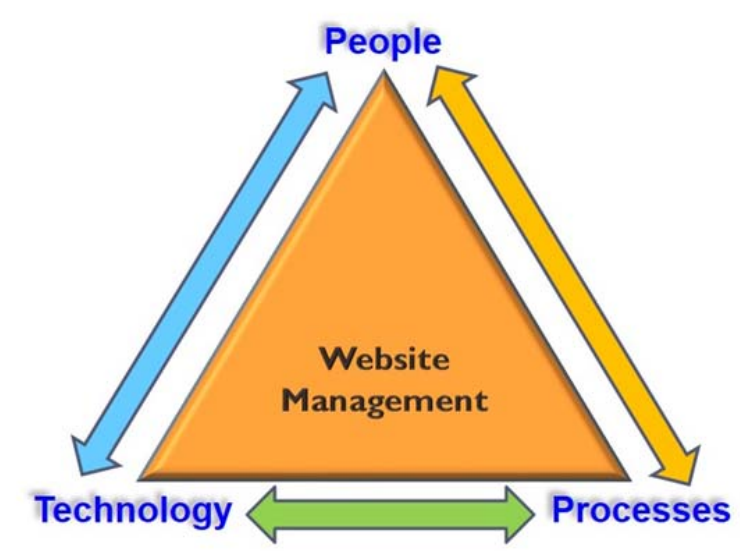

Figure 1. A holistic website management approach that is based on three key elements

- People: A multidisciplinary team structure with clearly defined leadership, roles, skills, and responsibilities is suggested to successfully manage university websites.

- Technology: A Content Management System (CMS) (e.g., the Drupal CMS in the GJU case) is identified as the technology of choice to develop non-interactive and content-based websites that contain rich UI components and require multiple collaborators to manage content.

- Processes: Suitable project management, software development, and website business processes are proposed and adopted to achieve organizational success and a high-quality website while meeting time and cost constraints.

The rest of the paper is organized as follows. In section 2, a comparison to related work is provided. In section 3, a possible website team structure is proposed. In section 4, the appropriate technology stack to develop a university website is suggested. In section 5, the aspects of a proper project management process are determined. In section 6, the software development process is identified. In section 7, the needed website business processes are discussed. Finally, a summary and conclusion are given in section 8 .

\section{Related Work}

As far as related work is concerned, the aim of the studies in [1],[2],[3] was to identify the website quality attributes that are most important to university stakeholders. Accordingly, it was found that website content, organization, appearance, navigation, usability, functionality, performance, security, and availability mostly aid in making a website a good marketing tool, and hence attract more students to enroll at a university [4]. The work in [5],[6],[7] discussed the development of university and library websites using the following CMSs: Drupal, Joomla, and WordPress. Whereas, the studies [8],[9],[10] utilized the PHP, ASP.NET, and Java Server Faces (JSF) frameworks to develop different dynamic websites. In the study in [11], the issue of improving the coordination between librarians and IT staff was investigated. In the works [12],[13], different criteria to select a CMS for website development were explored. In the study [14], a suitable development methodology was introduced to build high-quality websites.

However, based on the comparison shown in Table 1 , and unlike this work, the related papers did not address all the key elements of the proposed holistic approach to manage websites. Specifically, the project management process and the website business process were not covered by any of them. Besides, only three papers discussed team structure, though not in much depth as in this paper. It is also worth noting that we covered the details of the website content structure, design guidelines, development steps, content editing tasks, security schemes, and test types in the study in [15].

\section{Team Structure}

Maintaining a high-quality website requires a wellstructured multidisciplinary team with clearlydefined leadership, roles, responsibilities, and skills. A proposed web team structure that is suitable in a university setting is shown in Figure 2. Accordingly, the team members can be categorized into the following main groups:

- University Management Team: This team is usually led by the university president and typically includes vice presidents and school deans. It is mainly responsible for setting core business/marketing goals, approving website strategic plans, and monitoring adherence to plans.

- Web Committee: This committee is normally chaired by the IT director and includes representatives from all schools and departments with a stake in the website. Its mission is to specify and prioritize the desired website requirements, as well as set the website strategic plans when needed. In turn, the IT director presents any strategic plans to the university management team for approval and also passes any ratified requirements to the IT team for implementation. 
Table 1. Comparison to related work

\begin{tabular}{|c|c|c|c|c|c|c|}
\hline References & $\begin{array}{c}\text { Website } \\
\text { Quality } \\
\text { Attributes }\end{array}$ & $\begin{array}{c}\text { Website } \\
\text { Team } \\
\text { Structure }\end{array}$ & $\begin{array}{c}\text { Website } \\
\text { Development } \\
\text { Technology }\end{array}$ & $\begin{array}{c}\text { Website } \\
\text { Development } \\
\text { Process }\end{array}$ & $\begin{array}{c}\text { Project } \\
\text { Management } \\
\text { Process }\end{array}$ & $\begin{array}{c}\text { Website } \\
\text { Business } \\
\text { Processes }\end{array}$ \\
\hline This work & Yes & Yes & Yes & Yes & Yes & Yes \\
\hline$[1],[2],[3],[4]$ & Yes & No & No & No & No & No \\
\hline$[5]$ & Yes & Yes & Yes & No & No & No \\
\hline$[6],[9],[10],[12],[13]$ & Yes & No & Yes & No & No & No \\
\hline$[7],[8],[14]$ & Yes & No & Yes & Yes & No & No \\
\hline$[11]$ & No & Yes & No & No & No & No \\
\hline
\end{tabular}

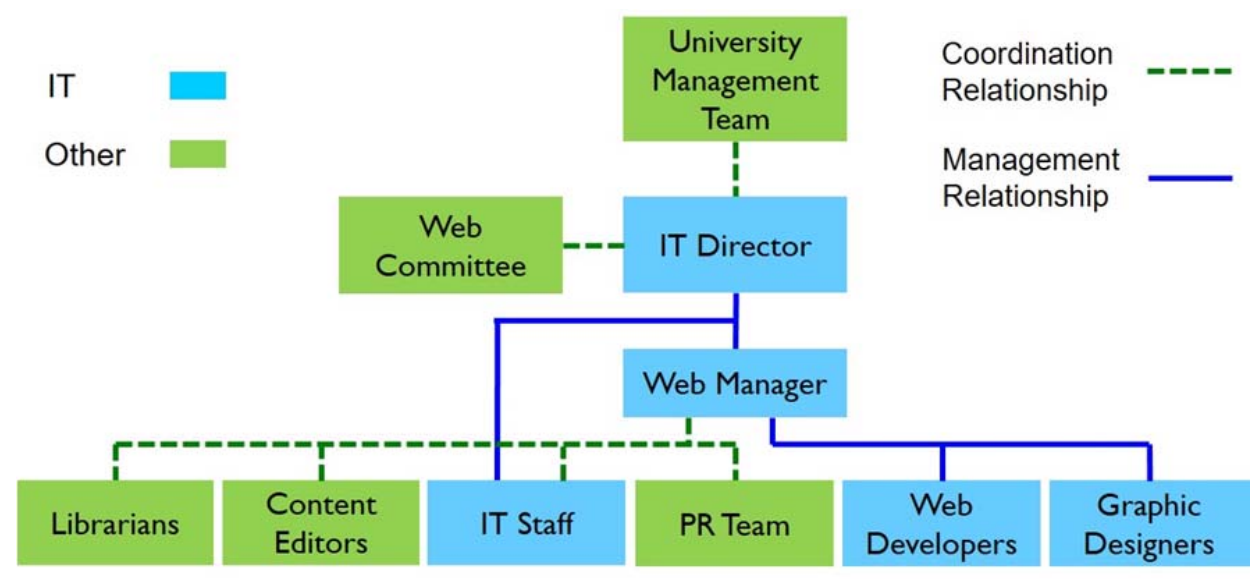

Figure 2. The structure of the university website team

- IT Center Team: The IT center team that is led by the IT director is considered the backbone of the web team structure since it is responsible for the technical, quality, and operational aspects of the website. Specifically, the website development team within the center is liable for administering, designing, developing, testing, and maintaining the website. It consists of web developers and graphic designers who are led by a web manager. The web manager further coordinates with the IT director, administers the operational tasks, collaborates with the related teams, trains the content editors, and checks the compliance of the published content with the website quality standards. On a related note, some required IT tasks (e.g., server administration, database administration, and website security) that are outside the scope of the website development team are usually delegated to other responsible teams (e.g., systems, databases, and networks teams) in the center.

- Public Relations Team: This team is responsible for publishing public content such as news, events, announcements; contact us about information, periodicals, photo galleries, etc. Mainly, it is comprised of content producers, photographers, and graphic designers.

- Content Editors: They are responsible for publishing the content of their respective academic or administrative entities. Therefore, they have to have excellent writing and editing skills, as well as work with the web manager closely to produce high-quality content that complies with the website standards.

- Library Team: This team is found in many universities to make the library e-resources, databases, portals, and search engines available on the website in coordination with the web development team. Also, this team is liable for editing the related content on the library subsite.

\section{Development Technology}

A website can be built using different types of web development technologies such as CMSs, as well as PHP, JSF, and ASP.NET frameworks. Based on that, selecting a technology depends on the suitability of its features with the application and business requirements. Appropriately, the website requirements are discussed first. Then, the features of common technologies are compared to suggest a proper tool and stack to develop a university website.

\subsection{Website Requirements}

It is important to emphasize that a website, in this context, is different than a web portal (e.g., help desk system [16]), and hence it can be characterized by the following basic features and requirements:

- Anyone can access the website from the internet without a login requirement. 
- Visitors cannot manipulate the published content.

- Content is dynamic, but not personalized for each visitor.

- Multiple collaborators (e.g., marketing, content editors, and librarians) from different schools and departments should be able to manage (add, edit, and delete) their respective content.

- Considered the first point of contact for university stakeholders.

- Content-based to inform visitors about the university brand, vision, values, objectives, strengths, services, news, programs, and achievements.

- Easy to use, engaging, and intuitive to navigate.

\subsection{Development Technology}

A comparison between CMSs and common web development frameworks is shown in Table 2 to identify a suitable technology for website development. Correspondingly, it is faster and easier to build a website using a CMS because most of the work can be performed through an administrative dashboard without the need for advanced programming and database design skills as required when frameworks are used. Further, the CMS allows installing various plugin modules to add UI features (i.e., menus, filters, galleries, maps, tabs, etc.) in the website pages. Besides, it allows assigning permissions as well as users to roles via a simple UI in order to support multiple users (i.e., web administrators, PR staff, and content editors) in a joint environment. To the contrary, the other frameworks are more flexible than the CMSs in case the web application requires integration with other systems and implementing complex workflows. However, the aforementioned disadvantage is negligible in this situation because the university website is simple and does not require such advanced features. The website is considered simple as it requires a flat structure, is not interactive, does not require authentication, does not personalize content, and does not demand complex workflows.

Accordingly, a CMS is the technology of choice to develop and maintain a university website. Specifically, the open-source Drupal CMS has been utilized to build the GJU website due to its costeffectiveness, security, performance, and large developer community [12],[13], as well as its popularity similarly to its counterparts Joomla and WordPress. In that regard, the fact that Drupal dynamically builds the website pages based on configuration and content information stored in the database, also eliminates the need to use modeldriven languages [17] like IFML (Interaction Flow Modelling Language) and WebML (Web Modelling Language) to automatically generate the website files. Still, entity-relationship (ER) and class diagrams can be used in this context to document the needed content types and structure.

With respect to the technology stack to utilize in this case, Drupal always runs on the open-source LAMP stack that is comprised of the Linux operating system, the Apache HTTP server, the MySQL database management system, and the PHP engine.

\section{Project Management Process}

Adopting a project management process is very important to produce a high-quality website while satisfying scope (e.g., functional requirements) along with time (e.g., schedule) and cost (e.g., budget) related constraints (see Figure 3). In that regard, the process that is based on the systems engineering basic profile in the ISO/IEC 29110 series [18] is appropriate to manage a small-size project intended to build a university website. Suitably, that process addresses important project related aspects like planning, progress, review, release, risks, and closure as it is shown in Figure 3 and discussed next.

Table 2. A comparison between CMSs and common web development frameworks

\begin{tabular}{|l|c|c|c|c|}
\hline & CMS & PHP Framework & JSF Framework & $\begin{array}{c}\text { ASP.NET } \\
\text { Framework }\end{array}$ \\
\hline $\begin{array}{l}\text { Provides an administration UI } \\
\text { panel }\end{array}$ & Yes & No & No & No \\
\hline Supports rich UI components & Yes & Not Easy & Yes & Yes \\
\hline Supports roles and permissions & Yes & No & No & No \\
\hline Flexible & No & Yes & Yes & Yes \\
\hline Faster development time & Yes & No & No & No \\
\hline Needs graphic design skills & Yes & Yes & Yes & Yes \\
\hline $\begin{array}{l}\text { Needs advanced programming } \\
\text { skills }\end{array}$ & No & Yes & Yes & Yes \\
\hline $\begin{array}{l}\text { Needs advanced database design } \\
\text { skills }\end{array}$ & No & Yes & Yes & Yes \\
\hline
\end{tabular}




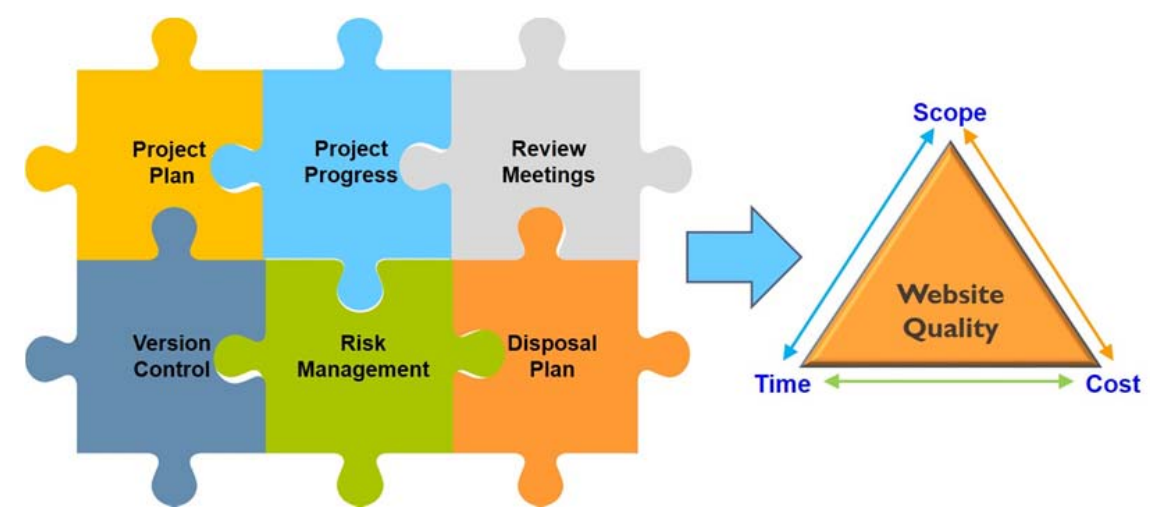

Figure 3. The main activities and advantages of the adopted project management process

\subsection{Project Plan}

A project plan is an important document that guides the management and execution of a project. In this context, the IT director is responsible for preparing the project plan and then presenting it to the university management team for approval. The project plan usually contains the project description, scope, users, deliverables, phases (i.e., the software development lifecycle phases), tasks (i.e., the tasks needed to complete all phases), schedules (i.e., the start and end dates for each phase and task), resources (i.e., headcount, skills, hardware, and software), costs (i.e., salaries, fees, training, travel, hardware, and software costs), and risks.

\subsection{Project Progress}

The IT director needs to release a monthly project progress report to the university management team and the web committee so they can monitor the project status. Specifically, the report should illustrate the phases/tasks progress (with respect to the related deadlines in the project plan), team assignment, accomplished milestones, and possible risks. Furthermore, the IT director is responsible for presenting the project status to the management team or web committee upon request. On the other hand, the developers and designers both submit weekly progress reports to the web manager for evaluation. In turn, the web manager discusses the project status with the IT director regularly to make sure everything is on track.

\subsection{Review Meetings}

Different types of review meetings are usually required to address various issues related to a project. For example, the development team typically conducts many sessions to review functional requirements, content structure, website design, test plans, and deployment steps. Besides, the development team may meet with the content editors to review the data entry guidelines. Moreover, the development team could demonstrate parts of an undergoing project to the web committee to solicit feedback and check its compliance with the desired requirements. In that regard, all meeting minutes have to be recorded and distributed to all attendees for feedback and approval.

\subsection{Version Control}

When a CMS is used to build a website, the site configuration and content are managed mostly using an administration dashboard. Consequently, all modifications are stored in a database rather than in files. Unfortunately, overwriting data in a database is mostly permanent and cannot be reverted easily to a previous state. Therefore, such changes cannot be tracked via typical version control systems such as Git. Accordingly, a suitable module has to be installed in the CMS for version control purposes. For example, the Features module can be installed in the Drupal CMS to allow modifying, updating, and reverting features.

\subsection{Risk Management}

A software project can be affected negatively by various risks that could be related to schedule (e.g., underestimated development time and unforeseen project scope), customers (e.g., changing requirements and resistance to change), developers (e.g., staff inadequacy, staff incompetence, and staff miscommunication), management (e.g., lack of support, change of managers, and change of priorities), and resources (e.g., insufficient budget, unavailability of hardware, and lack of software). Therefore, any encountered risks have to be managed efficiently to guarantee successful project completion. That can be achieved by monitoring the project details periodically to identify and resolve any risks promptly.

\subsection{Disposal Plan}


In case the new website is replacing an old one, a disposal plan has to be executed to address the following three concerns. Firstly, any needed content (e.g., text, images, videos, etc.) from the old website should be migrated to the new one. Secondly, the virtual machine, files, and database of the old website have to be archived on disk and tape for reference when needed. Thirdly, the old website should be brought down just moments before the launch of the new one to minimize the website downtime as much as possible.

\section{Development Process}

The six-phase website development process in [15] can be adopted to implement non-interactive websites. This process is interrelated with the aforementioned project management process as shown in Figure 4. Based on that, the development process is initiated when the project requirements as well as project plan are completed and then approved by the web committee. Suitably, the requirements are initially analyzed in phase 1 . Then, the web development team defines the content structure and designs the website templates in phase 2 and phase 3 , respectively. In phase 4 , the website is developed and the content (i.e., text, images, videos, etc.) is published. Subsequently, the website is launched and maintained in phase 5 and phase 6 , respectively. Noting that the details of all these phases are found in [15].

\section{Business Processes}

Adopting proper business-processes in a website division is very important to improve its productivity. In that regard, a business process is a set of work activities designed to produce a service for specific stakeholders. The processes and activities usually have owners (e.g., from the web team members) who are responsible for guaranteeing their successful execution. Therefore, optimizing (i.e., number of activities and time to complete every activity) and

ensuring the smooth implementation of the process activities contribute significantly to enhancing the effectiveness of an organization and thus improving customer satisfaction. In the next subsections, the website division main services, some business processes activities, as well as the business processes evaluation methods are identified and discussed.

\subsection{Website Division Business Processes}

Several business processes should be supported in the website division to offer all desired services and maintain the website quality. Ideally, stakeholders must be able to initiate a desired process, for handling by the website team, by opening a service ticket (see Figure 5) via a university helpdesk system (i.e., a software system used to manage IT services, user roles, and tickets [16]). In that regard, the proposed website division business processes are as follows:

- Website Creation Process: it is needed to add a new subsite (e.g., school, center, library, project, etc.) to the website.

- Content Update Process: it allows updating current content (e.g., adding an article to the news list, adding a record to the publications list, adding an image to a slideshow, etc.).

- Technical Issue Process: it allows reporting technical issues (e.g., website unavailability, performance issues, broken functionality, etc.) for handling by the website team.

- Quality Assurance Process: it enables scheduling website QA tests for broken links, spelling errors, grammatical mistakes, outdated content, guideline compliance, etc.

- Technical Report Process: it is required to request analytics and evaluation reports to evaluate the quality of the website and the productivity of the team.

- User Account Process: it deals with all issues related to user accounts such as addition, activation, deactivation, roles, passwords, etc.

- Editor Training Process: it is needed to request and schedule training sessions for current or new content editors.

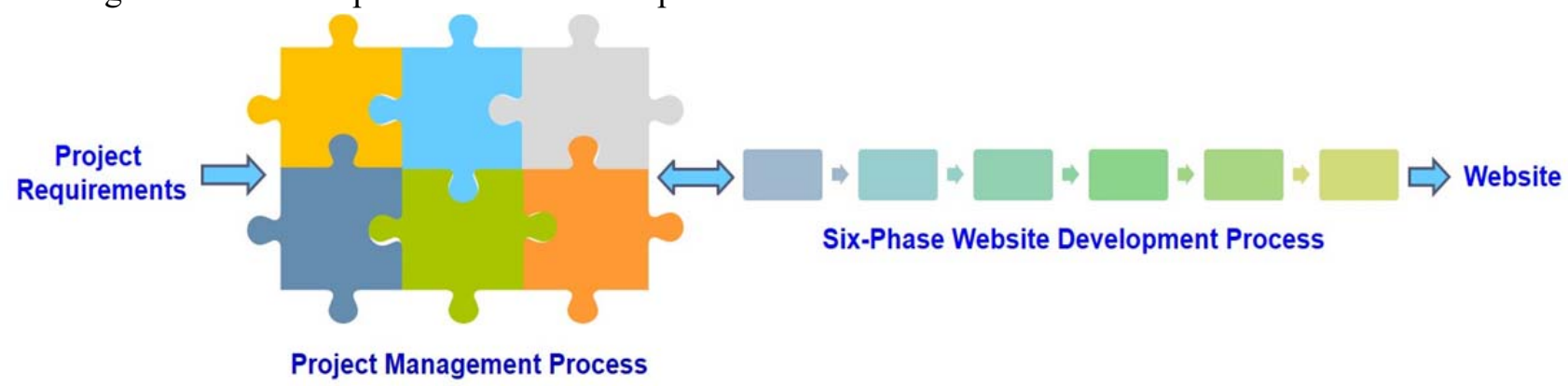

Figure 4. The interactions between the project management and development processes 


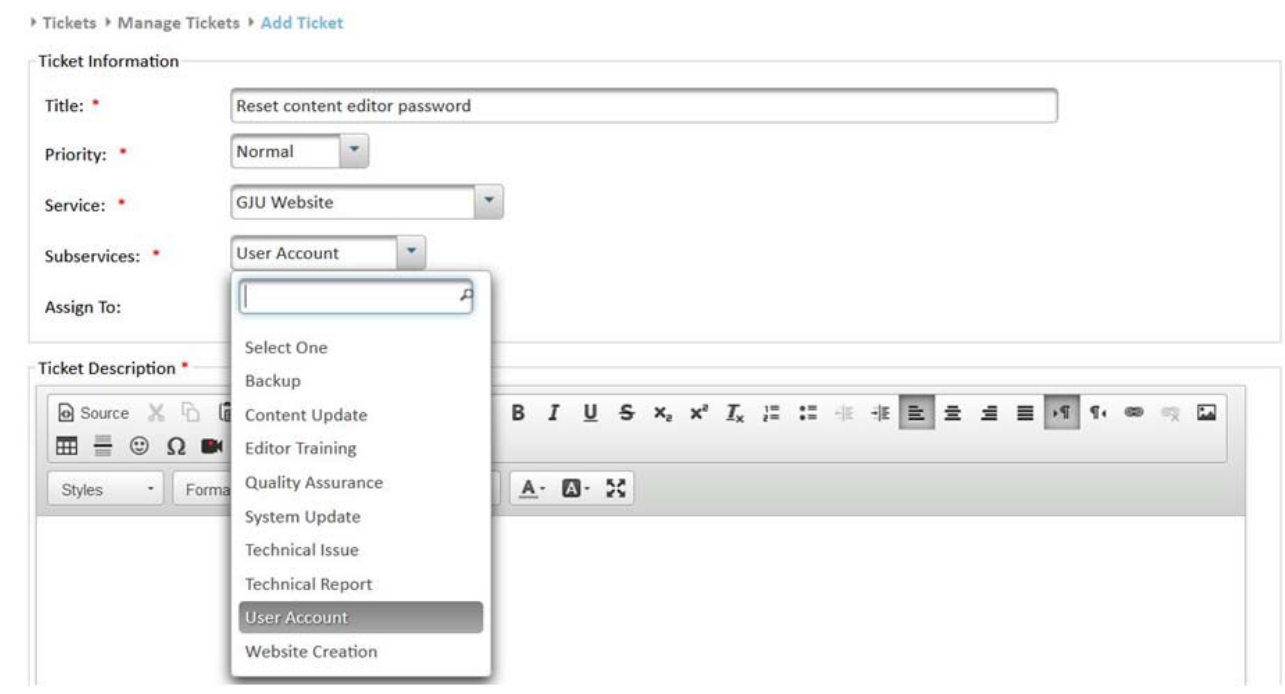

Figure 5. The supported website division business processes in the GJU helpdesk system [16]

- System Update Process: it deals with system updates such as updating Drupal, patching Linux, updating Apache, updating the antivirus software, etc.

- Backup Process: it allows scheduling a backup job for the website files or the machine image.

\subsection{Business Process Activities}

For illustration purposes, the activities of the aforementioned User Account Process and Technical Issue Process are shown in Table 3 and Table 4 respectively. Accordingly, in both cases, the requestor (i.e., the employee who is asking for the service) initiates the desired process by opening a service request ticket via the helpdesk system. When a ticket is opened, the website service admin (i.e., the employee responsible for managing the website service tickets, mostly the web manager) evaluates the request and then assigns the ticket to the responsible website service agent (i.e., the employee responsible for handling the assigned website service). Then, in activities 3, 4, 5, and 6 in the User Account Process, the agent performs the desired request (e.g., open a new account, close an existing account, reset password, etc.); tests the account; sends the account credentials (i.e., username and password) as well as the user guide to the requestor in case the account is new; and then updates and closes the ticket respectively. Correspondingly, in activities 3, 4, and 5 in the Technical Issue Process, the agent fixes the issue, tests the fix, and later updates and closes the ticket respectively. Accordingly, a business process focuses on the activities needed to offer a service and the parties responsible for the service delivery, rather than the details of what is being offered. Thus, it aids in evaluating the service delivery effectiveness.

\subsection{Business Process Evaluation Methods}

Identifying proper evaluation methods and key performance indicators (KPIs) is a very important managerial aspect to demonstrate the effectiveness of the adopted business processes and the quality of the offered services. In that regard, the utilized helpdesk system has to capture and produce useful data to measure various KPIs that are needed to evaluate the effectiveness of the website division. Examples on such KPIs are:

- Number of Tickets: A measure to find the total number of opened tickets during a selected period of time, the number of tickets opened against each service over a certain period of time, or the number of tickets that were assigned to each service agent over a certain period of time [16]. Thus, it helps in identifying the workload of the whole division, every business process, or each web service agent.

- Ratio of Closed to Overall Tickets: A measure of the percentage of closed to overall tickets for any service during a selected period of time [16]. Accordingly, the productivity of a business process is considered acceptable in that period when the value of this KPI becomes closer to $100 \%$.

- Average Ticket Assignment Time: A measure of the average ticket assignment time in a selected period of time [16]. Therefore, it demonstrates the responsiveness of the web team members to the various user requests.

- Average Ticket Resolution Time: A measure of the average ticket resolution time in a selected period of time [16]. Hence, it illustrates the duration it takes the web team members to successfully execute the required services. 
Table 3. The activities of the User Account Process

\begin{tabular}{|l|l|}
\hline \multicolumn{1}{|c|}{ Activity } & \multicolumn{1}{c|}{ Responsible Person } \\
\hline 1. A user account request is opened via the helpdesk system. & Requester \\
\hline 2. The request is evaluated and assigned to the responsible agent. & Website Service Admin \\
\hline $\begin{array}{l}\text { 3. The user account is managed as requested and the applicable permissions } \\
\text { are applied, if needed. }\end{array}$ & Website Service Agent \\
\hline 4. The account is tested. & Website Service Agent \\
\hline 5. The account credentials and user guide are sent to the requester, if needed. & Website Service Agent \\
\hline $6 . \quad$ The ticket is updated and closed. & Website Service Agent \\
\hline
\end{tabular}

Table 4. The activities of the Technical Issue Process

\begin{tabular}{|ll|l|}
\hline \multicolumn{1}{|c|}{ Activity } & Responsible Person \\
\hline 1. & A technical issue request is opened via the helpdesk system. & Requester \\
\hline 2. & The issue is evaluated and assigned to the responsible agent. & Website Service Admin \\
\hline $3 . \quad$ The issue is fixed. & Website Service Agent \\
\hline $4 . \quad$ The fix is tested. & Website Service Agent \\
\hline $5 . \quad$ The ticket is updated and closed. & Website Service Agent \\
\hline
\end{tabular}

Table 5. Sample questions to evaluate some of the website quality attributes

\begin{tabular}{|l|c|}
\hline \multicolumn{1}{|c|}{ Possible Question } & Measured Quality Attribute \\
\hline I often seek the website for new information & Engaging \\
\hline I can access the desired pages in a few clicks & Easy-to-use \\
\hline I think the website is organized & Structured \\
\hline I mostly find the needed content on the website & Content-rich \\
\hline I consider the website design attractive & Attractive \\
\hline I believe the website is fast to navigate & Fast \\
\hline I can visit the website anytime & Available \\
\hline
\end{tabular}

Nevertheless, other evaluation methods are also required to measure the KPIs that illustrate the website quality attributes. Accordingly, the following two evaluation methods are recommended:

a) Google Analytics: The university website can be linked to a google analytics account for traffic analysis and reporting purposes. Based on that, for example, it can be asserted whether a website is engaging, easy to use, compatible, available, and fast according to the data in the following reports: bounce rate (i.e., the percentage of users who navigate away from the website after viewing only one page), needed clicks to reach a page, used operating systems, users daily distribution, and page load time respectively.

b) User Surveys: Such surveys could be conducted over a specific period of time to evaluate the customer (e.g., staff, students, alumni, etc.) satisfaction regarding the website quality based on their answers to questions designed to measure the quality of particular attributes (see Table 5).

\section{Conclusion}

This work proposed a holistic approach to university website management based on three key elements: people, technology, and processes.

Accordingly, a multidisciplinary team can utilize an easy-to-use CMS to build a high-quality, contentbased, and non-interactive university website that fulfills the organizational requirements while satisfying the desired schedule and budget constraints.

The IT department is considered the backbone of the suggested web team structure. Suitably, the IT director heads a website committee to propose functional requirements and strategic plans for approval by a university management team. In turn, the development team builds the ratified features and then collaborates with multiple representatives from different organizational units to publish the related content. 
A website is considered different and less-complex than a web portal because it requires a flat structure, does not require login, does not allow a visitor to change content, and does not entail complex workflows. Therefore, a CMS is found more suitable to build university websites than other frameworks that require advanced coding and database skills.

A proper project management process based on the systems engineering basic profile in the ISO/IEC 29110 series was suggested to manage website related projects. This process covers important aspects such as project plan, progress, review, release, risks, and closure, which enable incorporating functional requirements with time and cost constrains. Furthermore, a six-phase website development process is deemed appropriate to implement simple and non-interactive websites.

Several business processes have been identified and adopted in the website division to offer the desired services, raise staff productivity, and achieve customer satisfaction. The processes are initiated and tracked via a helpdesk ticketing system to allow measuring KPIs needed for evaluating the effectiveness of the organization. Besides, google analytics and user survey based methods have been proposed to assess the quality attributes of the website.

\section{References}

[1]. Rennick, B. (2019). Library services navigation: Improving the online user experience. Information Technology and Libraries, 38(1), 14-26.

[2]. Owoyele S (2016) Website as a marketing communication tool. Thesis. Centria University of Applied Science, Kokkola, Finland.

[3]. Suwawi, D. D. J., Darwiyanto, E., \& Rochmani, M. (2015, May). Evaluation of academic website using ISO/IEC 9126. In 2015 3rd International Conference on Information and Communication Technology (ICoICT) (pp. 222-227). IEEE.

[4]. Ford, W. G. (2011). Evaluating the Effectiveness of College Web Sites for Prospective Students. Journal of College Admission, 212, 26-31.

[5]. Buell, J., \& Sandford, M. (2018). From Dreamweaver to Drupal: A University Library Website Case Study. Information Technology and Libraries, 37(2), 118-126.

[6]. Dutt, M., \& Kaur, B. (2016). Design and Development of University Library Website: A Case Study of Tagore Library, Chitkara University, Himachal Pradesh. Journal of Knowledge \& Communication Management, 6(2), 128-139.
[7]. Rab, S. (2018). A University website using Content Management System (CMS) and add-on plugins (Doctoral dissertation), United International University.

[8]. Kumari, P., \& Nandal, R. (2017). A Research Paper OnWebsite Development Optimization Using Xampp/PHP. International Journal of Advanced Research in Computer Science, 8(5).

[9]. Ren, J., Ding, H., \& Yang, X. (2010, April). Design and implementation of pedagogical courses teaching website based on ASP. In 2010 2nd IEEE International Conference on Information Management and Engineering (pp. 568-572). IEEE.

[10]. Naini, V. R., Sadasivam, R. S., \& Tanik, M. M. (2008, April). A Web-based interactive Student Advising system using Java frameworks. In IEEE SoutheastCon 2008 (pp. 172-177). IEEE.

[11]. Hoover, J. (2018). Gaps in IT and library services at small academic libraries in Canada. Information Technology and Libraries, 37(4), 15-26.

[12]. Mirdha, A., Jain, A., \& Shah, K. (2014, December). Comparative analysis of open source content management systems. In 2014 IEEE international conference on computational intelligence and computing research (pp. 1-4). IEEE.

[13]. Patel, S. K., Rathod, V. R., \& Prajapati, J. B. (2011). Performance analysis of content management systems-joomla, drupal and wordpress. International Journal of Computer Applications, 21(4), 39-43.

[14]. Yahaya, J. H., Ibrahim, A. A., \& Deraman, A. (2017). Software process model for dynamic website development towards quality product. Journal of Telecommunication, Electronic and Computer Engineering (JTEC), 9(3-3), 39-44.

[15]. Al-Hawari, F., Al-Zu'bi, M., Barham, H., \& Sararhah, W. The GJU Website Development Process and Best Practices. Journal of Cases on Information Technology (JCIT), 23(1), 21-48.

[16]. Al-Hawari, F., \& Barham, H. (2019). A machine learning based help desk system for IT service management. Journal of King Saud UniversityComputer and Information Sciences. doi:https://doi.org/10.1016/j.jksuci.2019.04.001

[17]. Rossi, G., Urbieta, M., Distante, D., Rivero, J. M., \& Firmenich, S. (2016). 25 Years of Model-Driven Web Engineering. What we achieved, What is missing. CLEI Electronic Journal, 19(3), 5-57.

[18]. Laporte, C. Y., O’Connor, R. V., \& Paucar, L. H. G. (2015, April). The implementation of ISO/IEC 29110 software engineering standards and guides in very small entities. In International Conference on Evaluation of Novel Approaches to Software Engineering (pp. 162-179). Springer, Cham. 